\title{
La logique du raisonnement de précaution
}

Mark Hunyadi

\section{OpenEdition}

\section{Journals}

Édition électronique

URL : http://journals.openedition.org/ress/341

DOI : 10.4000/ress.341

ISSN : 1663-4446

\section{Éditeur}

Librairie Droz

Édition imprimée

Date de publication : 1 novembre 2004

Pagination : 9-33

ISBN : 2-600-00980-9

ISSN : 0048-8046

Référence électronique

Mark Hunyadi, «La logique du raisonnement de précaution », Revue européenne des sciences sociales [En ligne], XLII-130 | 2004, mis en ligne le 03 novembre 2009, consulté le 19 avril 2019. URL : http:// journals.openedition.org/ress/341 ; DOI : 10.4000/ress.341 
Mark HUNYADI

\section{LA LOGIQUE DU RAISONNEMENT DE PRÉCAUTION}

\section{MATRICE ET PORTÉE DU PRINCIPE DE PRÉCAUTION}

Une seule chose est certaine, à propos du principe de précaution (PP) - qui est précisément en charge de nous orienter dans des situations d'incertitude -, c'est que son entrée en scène publique s'est effectuée en double détente: au Sommet de la Terre à Rio en juin 1992, où il a été universellement élevé à la dignité de superou méta-principe (sans pourtant qu'il ait une force juridique contraignante) servant à définir un nouveau type de rapport entre les hommes et leur environnement; à l'occasion de la crise de la vache folle ensuite, qui, dans une ambiance de branle-bas de combat, en a fait l'indispensable référence de toute politique de sécurité alimentaire, élargissant du même coup sa validité à d'autres domaines qu'environnementaux. Mais à vrai dire, même sur ce dernier point, les interprétations relatives au principe de précaution divergent largement: si l'on s'accorde généralement à penser que l'affaire de la vache folle illustre bel et bien «la nonapplication du principe de précaution $»^{1}$, les opinions diffèrent sur l'efficacité réelle qu'aurait pu avoir son application aux différents niveaux de la crise de l'ESB. Pour Corinne Lepage, il ne fait pas de doute par exemple que «les choses auraient pu être différentes s'il avait joué», suivant en cela d'ailleurs le rapport Viney-Kourilsky ${ }^{2}$; et de détailler les violations successives du principe de précaution depuis décembre 1987, où ont été établis les premiers liens entre l'ESB et les farines animales utilisées comme compléments alimentaires. En revanche, pour Godard, Henry, Lagadec et Michel-Kerjan, «l'adoption précoce du principe de précaution n'aurait pu empêcher ni le scénario du développement de l'épidémie qu'on tenait pour acquis avant le rapport Phillips, ni le scénario réel du développement de cette épidémie $»^{3}$. C'est dire que l'utilisation du principe de précaution est elle-même sujette à bien des incertitudes...

Historiquement, avant d'être invoqué dans la Déclaration de Rio en 1992 et dans le Traité de l'Union européenne (Maastricht) la même année, il a fait sa première apparition en droit international dans la Déclaration de Londres en

Corinne Lepage et François Guéry, La politique de précaution, Paris, Seuil, 2001, p. 111.

Geneviève Viney et Philippe Kourilsky, Le principe de précaution, Rapport au premier ministre, Paris, Odile Jacob, 1999, annexe.

Olivier Godard, Claude Henry, Patrick Lagadec, Erwann Michel-Kerjan, Traité des nouveaux risques, Précaution, risque, assurance, Paris, Gallimard, coll. Folioactuel, 2002, p. 112. (Cidessous: TNR). 
$1987^{4}$, adoptée par la deuxième Conférence sur la mer du Nord, où les ministres «acceptent que, afin de protéger la mer du Nord des effets dommageables éventuels des substances les plus dangereuses, une approche de précaution est nécessaire, qui peut requérir l'adoption de mesures de contrôle de ces substances avant même qu'un lien de cause à effet soit formellement établi sur le plan scientifique $»^{5}$. Depuis ces textes inauguraux, il a été invoqué dans de très nombreuses conventions internationales, parmi lesquelles la Convention sur la diversité biologique (1992), une «convention-cadre » qui pose des principes généraux (en réaffirmant notamment la souveraineté des États sur leurs ressources) et qui a donné lieu au Protocole de Cartagena sur la prévention des risques biotechnologiques (Montréal, 29 janvier 2000), qui est en fait un traité de commerce international réglementant les échanges d'OGM ${ }^{6}$ (essentiellement les semences). L'article $1 \mathrm{du}$ Protocole stipule: "Conformément à l'approche de précaution consacrée par le Principe 15 de la Déclaration de Rio sur l'environnement et le développement, l'objectif du présent Protocole est de contribuer à assurer un degré adéquat de protection pour le transfert, la manipulation et l'utilisation sans danger des organismes vivants modifiés résultant de la biotechnologie moderne qui peuvent avoir des effets défavorables sur la conservation et l'utilisation durable de la diversité biologique, compte tenu également des risques pour la santé humaine, en mettant plus précisément l'accent sur les mouvements transfrontières ${ }^{7}$. Par où l'on voit que très vite, le PP a débordé le cadre strictement environnemental qui était le sien au départ, pour s'appliquer au domaine sanitaire (santé publique), au domaine de la sécurité alimentaire et des manipulations génétiques: une extension qui assurément nuit à la précision du concept tout en banalisant son usage, notamment dans le discours politique où il est omniprésent ${ }^{8}$.

Que dit au juste le principe de précaution? Dans la jungle de ses innombrables formulations, une idée centrale se dégage que l'on peut synthétiser comme suit: lorsqu'il y a présomption raisonnable d'un risque déraisonnable, l'absence de certitude scientifique quant à la réalisation de ce risque ne doit pas être prétexte à retarder l'adoption de mesures visant à limiter ou à éliminer ce risque. C'est ce

Pour une description détaillée de l'émergence du principe de précaution, voir Pascal van Griethuysen, Le Principe de précaution: quelques éléments de base, Les cahiers du RIBios $\mathrm{n}^{\circ} 4$, RIBios-IUED, Genève, mars 2004.

5 Cité dans François Ewald, Christian Gollier, Nicolas de Sadeleer, Le principe de précaution, Paris, P.U.F., coll. Que sais-je?, 2001, p. 8. Au niveau national, le principe de précaution figurait déjà dans les Directives sur la précaution en matière d' environnement que le gouvernement allemand a adopté en 1986. Ce même gouvernement mentionnait toutefois la «vorsorgende Umweltpolitik» dès 1976. Ibid, pp. 6-7.

$6 \quad$ Plus exactement, aux OVM, c'est-à-dire aux organismes vivants modifiés, ce qui constitue un sousensemble des OGM: les graines génétiquement modifiées sont des OVM, mais les farines qui en résultent sont des OGM. Les aliments transformés ne sont pas des OVM, et ne tombent donc pas sous le coup du Protocole. Pour une discussion du Protocole, voir Philippe Cullet, The Biosafety Protocol: an Introduction, IELRC Briefing Pape 2002-2: www :ielrc.org/Content/ BP02012 P.pdf

Protocole de Cartagena sur la prévention des risques biotechnologiques relatif à la convention sur la diversité biologique, Montréal, 2000, p. 3

On trouvera une chronologie complète des normes aux niveaux français et européen dans Suzanne de Cheveigné, Daniel Boy et Jean-Christophe Galloux, Les biotechnologies en débat, pour une démocratie scientifique, Paris, Balland, 2002, pp. 221 à 251. 
que dit la formulation canonique de Rio: «En cas de risques de dommages graves ou irréversibles, l'absence de certitude scientifique absolue ne doit pas servir de prétexte pour remettre à plus tard l'adoption de mesures effectives visant à prévenir la dégradation de l'environnement». La Loi Barnier du 2 février 1995 ajoute à cette formulation l'idée de proportionnalité (sur laquelle nous reviendrons) et de coûts économiques, mais repose sur le même principe: «L'absence de certitudes, compte tenu des connaissances scientifiques et techniques du moment, ne doit pas retarder l'adoption de mesures effectives et proportionnées visant à prévenir un risque de dommages graves et irréversibles à l'environnement à un coût économiquement acceptable ${ }^{9}$. En ajoutant ici la référence aux coûts économiques des mesures envisagées, la Loi Barnier semble en réalité plus proche de la formulation originelle anglaise de la Déclaration de Rio, puisque celle-ci mentionnait des mesures «cost-effective», là où le français n'a retenu que les «mesures effectives »: «costeffective» veut dire que l'on escompte explicitement des mesures prises qu'elles le soient au coût le plus bas possible pour l'efficacité la plus haute, alors que les mesures «effectives » ne sont que des mesures concrètes ou tangibles dont la seule exigence est en l'occurrence d'éviter de trop grandes dégradations de l'environnement. Comme la formulation originaire de Rio, la Loi Barnier réinscrit donc l'application du PP dans la logique du calcul coût/bénéfices, sans pour autant faire de ce calcul le critère dominant de la décision: celle-ci reste toujours fondamentalement ordonnée à l'évitement de conséquences déraisonnables.

Quelle que soit donc la formule adoptée, il semble qu'elle se décline toujours sur cette matrice fondamentale de l'injonction à éviter des conséquences négatives trop grandes dans un contexte d'incertitude scientifique. Pierre Lascoumes l'exprime sous forme d'adage: «Dans le doute sur l'existence et la portée d'effets potentiellement négatifs, sur l'homme ou l'environnement, d'un produit ou d'une activité, ne t'abstiens surtout pas. Engage au contraire une démarche d'évaluation du danger et de recherche des moyens de le maîtriser ${ }^{10}$, si l'on précise toutefois que la recherche de ces moyens doit se faire à un coût économiquement acceptable. On ne saurait de ce point de vue sous-estimer la portée du principe de précaution, considéré dans son rapport aux activités techniques en général. Dans son intention, il entend en effet fournir un principe de régulation à toute une sphère de l'activité des hommes, à savoir les activités instrumentales qui déploient leur emprise scientifique, technique, industrielle et commerciale sur le monde. Il s'agit en effet d'imposer - si possible par le droit, mais à tous le moins dans les intentions - une limite de principe aux activités instrumentales, qui, livrées à elles-mêmes, suivent une logique simplement cumulative et orientée en fonction d'une fin qu'elles établissent elles-mêmes; alors qu'elles sont par leur nature même rationnelles en fonction d'une fin, elles sont à présent en quelque sorte sommées de devenir responsables à l'égard des conséquences provoquées en dehors de leur sphère d'action particulière. Ce qui est visé en effet, ce sont essentiellement les retombées collectives des activités instrumentales, et notamment les retombées sur des acteurs sociaux qui ne sont, eux, en rien responsables de l'acti-

9 Textes cités dans Olivier Godard (dir,), Le principe de précaution dans la conduite des affaires humaines, Paris, éd. de la Maison des sciences de l'homme, 1997, p. 43.

10 Pierre Lascoumes, «Le principe de précaution», in: Encyclopaedia Universalis sur CD-Rom, version 8, 2003. 
vité instrumentale en question. Pour une entreprise, un risque de faillite à la suite de la mise sur le marché quelque peu aléatoire de l'un de ses produits n'est pas, pour cette raison, un risque qui tombe sous le coup de la précaution, au contraire des externalités que sont des dommages environnementaux, des atteintes à la sécurité alimentaire ou à la santé publique. Les risques à l'égard desquels les acteurs d'activités instrumentales sont sommés de devenir responsables sont donc des dommages collectifs qui dépassent la sphère d'activités propre de l'activité instrumentale considérée.

De ce point de vue, la traduction de cette exigence de responsabilité dans les textes juridiques internationaux puis nationaux marque peut-être un véritable tournant dans le développement de nos sociétés, puisqu'il entend explicitement limiter, pour la première fois, le développement abandonné à lui-même de la rationalité instrumentale telle qu'elle s'exprime prioritairement dans la maîtrise technique de la nature et de l'homme lui-même. Car jusque là valait implicitement l'axiome: ce qui est globalement bon pour le développement de la rationalité instrumentale est globalement bon pour l'homme; et les éventuels dégâts causés par les développements technologiques seront à leur tour corrigés par la science elle-même. Le principe de précaution apparaît ainsi comme le premier outil public et contraignant (car comme le rappelait la résolution du Conseil européen de Nice en 2000, sa mise en œuvre est du ressort des « autorités publiques ») qui brise cette logique du tout-instrumental, en établissant explicitement un ordre de priorité qui le limite: le développement, oui, mais de telle manière qu'aucune action ne vienne mettre en péril les conditions de vie des générations actuelles et futures. C'est la raison pour laquelle le principe de précaution est aussi à comprendre dans la perspective plus large du développement durable au sens que lui donnait le rapport Brundtland (1987), autrement dit d'un développement qui doit «répondre aux besoins du présent sans compromettre la capacité des générations futures à répondre à leurs propres besoins». Le PP est destiné à restituer la priorité d'un environnement collectif (écologique, climatique, sanitaire, alimentaire) sain face aux dangers, mêmes incertains, qui le menacent. Il se donne pour objectif clair d'éviter des dangers trop grands, fût-ce au détriment de la logique du développement économique et industriel, même si les mesures ellesmêmes, comme nous l'avons vu, doivent elles aussi obéir à une logique d'efficacité économique. La préservation de la vie, quoi qu'on entende exactement par là, est devenue, par l'intermédiaire du principe de précaution, globalement prioritaire. C'est pourquoi le $\mathrm{PP}$ a, en tant que principe appliqué aux activités instrumentales de portée collective, une indéniable portée sociétale.

\section{LES ÉCOLES DE LA PRÉCAUTION}

En tant qu'outil juridique, le principe de précaution enjoint donc de ne pas s'abstenir d'agir à l'encontre d'une action envisagée du simple fait que le risque qu'elle nous fait encourir n'est pas scientifiquement certain. Cette formulation comme d'ailleurs l'adage de Pierre Lascoumes le dit explicitement - rend à mon sens inutile la discussion oiseuse sur la question de savoir si le principe de précaution est un principe d'action ou d'abstention: certains de ses critiques prétendent en effet que le PP conduit à une inaction généralisée, parce que paralysant toute initiative novatrice, par définition risquée. Critique à l'évidence de mauvaise foi : 
car s'il peut effectivement s'agir de s'abstenir de certaines actions jugées potentiellement trop dangereuses, il s'agit avant tout d'agir, mais d' 'agir dans un monde incertain », comme le dit le dit le titre d'un livre sur la question ${ }^{11}$, c'est-àdire d'agir dans un cadre qui soumet l'action à de nouveaux critères d'évaluation. En outre et plus fondamentalement encore, le PP rigoureusement appliqué pourrait bien être un formidable moteur de la connaissance scientifique elle-même, puisqu'il incite à explorer des solutions alternatives à celles jugées trop risquées, à ouvrir des chemins non encore balisés. Le PP est bien plus générateur qu'inhibiteur de connaissance, et il n'est pas absurde de penser qu'il est l'un des meilleurs leviers de l'extension de la diversité technologique.

Mais au-delà de cette querelle sans grand intérêt (car si vraiment le PP ne visait qu'à paralyser l'action, il ne permettrait effectivement d'affronter aucun des grands défis de notre temps), le principe de précaution a donné lieu à des interprétations diverses, qui sont autant d' «écoles de la précaution». On peut en donner la classification approximative suivante, qui recouvre à chaque fois une version différente de la nature de la précaution ${ }^{12}$ :

- l'école catastrophiste, qui ordonne le PP en fonction du risque maximal envisageable, c'est-à-dire la destruction de l'humanité (Hans Jonas, Jean-Pierre Dupuy);

- l'école prudentielle, qui fait de la précaution la version moderne de la prudence traditionnelle, inhérente à toute action envisagée (Philippe Kourilsky, Geneviève Viney);

- l'école dialogique, qui fait de la précaution le levier de la démocratie participative en général, en mettant l'accent sur les procédures communicationnelles qui doivent être mises en œuvre pour aboutir, dans la gestion de l'incertain, à des décisions acceptables par tous (Michel Callon, Pierre Lascoumes, Yannick Barthe).

\section{a. L'école catastrophiste}

Très critiquée, elle a néanmoins une incontestable dignité, puisqu'elle peut se réclamer du père fondateur de la précaution, le philosophe Hans Jonas (19031993) qui dans son œuvre maîtresse, Le principe responsabilité ${ }^{13}$, a formulé de la

${ }^{11}$ Michel Callon, Pierre Lascoumes, Yannick Barthe, Agir dans un monde incertain, essai sur la démocratie technique, Paris, Seuil, coll. La couleur des idées, 2001. Dorénavant: AMI.

12 Un autre fil conducteur donnerait une autre classification. Ainsi par exemple, on peut classer les versions du PP non selon l'interprétation qu'elles donnent de la précaution elle-même, mais selon la force normative qu'on lui accorde, comme le fait dans ce volume Pascal van Griethuysen, «Pour une approche évolutive de la précaution», qui suit en cela Dominique Bourg. On obtient alors la classification suivante: 1/ L'approche de précaution: recommandation d'une ligne de conduite générale à adopter dans le domaine environnemental, sans contrainte juridique; 2/ PP comme principe juridique de politique publique, qui donne aux États la capacité d'intervenir au nom de la précaution à l'encontre d'autres intérêts, p.ex. industriels; 3/ PP comme paradigme général d'action, qui obligerait les États et les acteurs sociaux à contrôler l'essor de toutes les techniques. Pour Pierre Lascoumes, le PP représente ce que les juristes appellent un «standard de jugement »: voir Pierre Lascoumes, «La précaution : un nouveau standard de jugement» in Esprit, novembre 1997, p. 129. On trouvera une analyse de la portée normative du PP notamment dans Corinne Lepage, François Guery, La politique de précaution, Paris, P.U.F., 2001, pp. 108-111. 
façon la plus profonde et la plus radicale l'exigence morale d'assurer la survie des générations futures - ce qu'il appelle d'une manière générale le maintien de la propre présupposition du principe de responsabilité, c'est-à-dire que chaque responsabilité, «à côté de ses tâches particulières, comporte également la responsabilité que par-delà son propre accomplissement subsiste encore la possibilité d'un agir responsable dans l'avenir ${ }^{14}$. Il donne à cette exigence morale la forme d'un impératif calqué sur l'Impératif catégorique de Kant: "Agis de façon que les effets de ton action soient compatibles avec la permanence d'une vie authentiquement humaine sur terre », qu'il formule aussi négativement: "Agis de façon que les effets de ton action ne soient pas destructeurs pour la possibilité future d'une telle vie »; plus simplement: «Ne compromets pas les conditions pour la survie indéfinie de l'humanité sur terre»; ou encore: «Inclus dans ton choix actuel l'intégrité future de l'homme comme objet secondaire de ton vouloir $\gg^{15}$. Formuler une telle exigence, qui déborde le cadre de toutes les éthiques traditionnelles, des éthiques de proximité qui ne pouvaient pas du tout imaginer que la nature pût en tant que telle devenir un objet de responsabilité morale, formuler une telle exigence donc est devenu nécessaire du fait même de la nouvelle relation technologique que l'homme, à l'âge nucléaire, a établi avec la nature.

On comprend dans cette perspective que Jonas accorde une prééminence dans son système à la "prophétie de malheur $»^{16}$ et à ce qu'il désigne explicitement de «la priorité du mauvais pronostic sur le bon ${ }^{17}$ : «l'axiome de base » étant «qu'il doit y avoir un avenir ${ }^{18}$, tout est ordonné à la destruction possible du monde humain ou humainement habitable. Si dans les petites actions sans conséquence, on peut se permettre des coups manqués dans l'espoir de quelque réussite, il n'en va pas de même pour les actions dont les conséquences sont possiblement fatales: il faut les éviter à tout prix. De plus, la logique cumulative du développement technologique doit nous rendre particulièrement attentifs aux commencements, car très vite, ces développements s'autonomisent et deviennent progressivement irréversibles (que l'on songe, par exemple, à ce qu'impliquerait pour nous un renoncement à l'automobile, une invention peut-être fatale...). Quoi qu'il en soit, si la perspective de Jonas peut à bon droit être taxée de catastrophiste, elle a néanmoins de bonnes raisons pour elle. Ses détracteurs se focalisent pourtant sur cette thèse de la prééminence du scénario du pire pour dénoncer chez l'auteur du Principe responsabilité je ne sais quelle régression antimoderne, irrationaliste et hostile à la science ${ }^{19}$. Rien n'est plus faux. On lui reproche son prétendu irrationalisme à cause de son heuristique de la peur, une peur dont on pense qu'elle est

13 Hans Jonas, Le principe responsabilité, une éthique pour la civilisation technologique, trad. de Jean Greisch, Paris, éd. du Cerf, 1990. (Ci-dessous: PR). L'original allemand (Das Prinzip Verantwortung) a paru en 1979.

14 PR 165.

15 PR 30-31.

$16 \quad$ PR 58.

$17 \quad$ PR 54.

$18 \quad$ PR 48.

19 Ainsi par exemple chez Catherine Larrère, «La nature à l'épreuve des nouvelles mutations », in: Les grandes mutations, 38 Rencontres Internationales de Genève, Lausanne, éd. L'Age d'homme, 2001, notamment p. 166. Voir aussi le compte-rendu de ma discussion avec elle dans le 
mauvaise conseillère en démocratie. Mais la peur dont parle Jonas n'est pas la peur paralysante qui inhibe l'action; c'est une heuristique au contraire, c'est-àdire un instrument de connaissance, une peur qui révèle, au sens photographique du terme, que quelque chose doit être pensé ou repensé; elle n'est que le prélude à la réflexion ou à l'action réfléchie, un prologue à la prudence. Quant à son antimodernisme et sa prétendue hostilité à la science, il n'est que de lire la fin de son livre (il est vrai difficile) pour se convaincre du contraire; sous le chapitre «Le progrès avec précaution», on peut lire: «Rien de ce qui précède ne devrait être mésinterprété comme si l'on déconseillait tel ou tel progrès technique, même si le caractère périlleux des cadeaux de ce pouvoir entre les mains de l'avidité et de l'étroitesse de vue humaines (et même de la misère) est un thème récurrent de notre mélodie. La fusion nucléaire, si jamais nous en sommes comblés, pourrait résoudre le problème de l'énergie à jamais. Seulement le cadeau doit être utilisé sagement et avec mesure, dans l'optique de la responsabilité globale et non dans celle de la grandiose espérance globale. Il faudrait d'abord calculer où se situerait la limite de la nature, ou bien où commencerait le seuil de danger critique, à supposer qu'on parvienne à l'embarras de la richesse. Mais cela est nécessaire longtemps avant pour les facteurs actuels dont nous avons effleuré quelques-uns (comme le sort biochimique du sol et des nappes phréatiques, l'économie de l'oxygène planétaire, et ainsi de suite), dont il vaudrait mieux anticiper les seuils critiques plutôt que de leur permettre d'en apporter la démonstration par leur réalisation même $»^{20}$.

Dans la ligne de Jonas, Jean-Pierre Dupuy, héraut du rationalisme contemporain, retient pourtant lui aussi l'idée «catastrophiste» selon laquelle la simple possibilité de la catastrophe justifie que l'on retienne le scénario du pire: approuvant (et admirant) explicitement Jonas, il note contre ses critiques qu' «il est rationnel aujourd'hui d'être catastrophiste dans le sens indiqué par le commandement de Jonas $»^{21}$. Deux points, si je vois bien, le distinguent ou plutôt le démarquent de son grand prédécesseur: d'une part - et cela est moins important pour nous ici -, il conteste que la technique soit par elle-même autonome, comme le laisse entendre Jonas. Si elle l'est - et elle l'est -, ce n'est pas par une force destinale qui s'imposerait à nous à la manière grecque ou heideggérienne, c'est par des mécanismes mis en œuvre par les acteurs sociaux, principalement des mécanismes d'imitation qui par rétroaction positive produisent des effets de renforcement: pour Dupuy, la technique n'est pas par essence autonome, elle le devient par la force mythopoïétique de l'imitation généralisée ${ }^{22}$. Mais c'est surtout le second point qui est au centre de ses réflexions sur la précaution: alors que Jonas consacre la plus grande partie de son à fonder l'impératif de survie, Dupuy, lui, s'intéresse surtout à fonder sa crédibilité: «On ne croit à l'éventualité de la catastrophe qu'une fois celle-ci advenue, telle est la donnée de base $»^{23}$. Ce qui retient donc Dupuy, c'est la capacité, ou plutôt l'incapacité du scénario du pire à mobi-

même volume, pp. 180-181. Voir aussi dans le même sens les pages qui lui sont consacrées dans le Traité des nouveaux risques de Godard, Henry, Lagadec et Michel-Kerjan (2002), pp. 82-93.

20 PR 256-257.

21 Jean-Pierre Dupuy, Pour un catastrophisme éclairé, Paris, Seuil, 2002, p. 93 (Dorénavant: PCE).

22 Pour ce thème, voir PCE 65-77.

23 PCE 169. 
liser l'action. Le statut ontologique de la catastrophe est le statut d'un possible non réalisé: un statut trop faible pour provoquer (quasi causalement) la croyance en sa vérité. Son problème est donc de croire à la réalité de la catastrophe, ce que reflète le titre de son chapitre «Savoir n'est pas croire», dont le leitmotiv est de dire: le problème du PP n'est pas de gérer l'incertain, mais d'agir sur l'incrédulité, car nous ne croyons pas à la catastrophe inéluctable; il y a impossibilité de croire que le pire va arriver ${ }^{24}$. L'annonce des catastrophes ne produit aucun changement sensible $^{25}$ : ainsi l'annonce du réchauffement climatique ou de la diminution de la couche d'ozone (on a ainsi vu G. W. Bush renoncer à réduire l'émission des gaz à effet de serre). L'incertitude est un alibi, parce que croire à la catastrophe aurait de telles répercussions sur nos croyances antérieures que nous préférons ne pas les bouleverser, par où se manifeste le besoin humain de maintenir la cohérence des croyances. Dans un réflexe dénégateur, l'esprit écarte ce qu'il a peur de croire.

La réponse à cette difficulté, réponse par laquelle Dupuy entend combler une lacune dans la pensée de Jonas (qui ne se pose jamais la question de savoir comment motiver l'esprit à agir en fonction du jugement qui a été fondé), consiste à renverser la métaphysique traditionnelle du temps - ce qu'il appelle «le temps de l'histoire»-, qui tient le passé pour fixe et le futur pour possible, au profit du « temps du projet», en coordonnant l'action autour d' un avenir tenu pour fixe, de manière à ce que «la réaction à l'avenir anticipé boucle causalement sur l'anticipation en question ${ }^{26}$. Comment faire? Le problème de Dupuy est de prédire pour que la prédiction n'advienne pas, ce qui est très différent de ce qu'on pourrait appeler «le paradoxe de Madame Soleil», qui fait qu'une prédiction s'est révélée fausse parce qu'elle avait été faite. Ainsi, si vous renoncez à prendre votre voiture parce qu'on vous a prédit un accident ce jour-là, la prédiction s'est révélée fausse du fait même que vous en avez tenu compte. Mais ici, il s'agit d'autre chose: le prophète, conscient de l'influence de sa prédiction, prédit l'avenir pour qu'il ne se produise pas. C'est sans doute cette conscience réflexive qu'il appelle «la lucidité du prophète». Mais là encore, comment faire pour que cet avenir tenu fixe provoque causalement son évitement? D’apories en apories, la solution, sibylline, nous est révélée. Elle est tirée des théories de stratégie de dissuasion nucléaire, et consiste à dire que l'impossible doit être tenu pour impossible, à un accident

\section{PCE 142-143.}

25 Dupuy ne fait aucune allusion au fait que cette question est la version sophistiquée d'un problème très classique, celui de la faiblesse de la volonté, identifiée par Aristote comme akrasia. L'échelle est certes différente (notamment parce qu'il s'agit ici de volonté collective), mais fondamentalement, ne s'agit-il pas ici du fait que l'on n'agit pas en fonction de son meilleur jugement?

26 PCE 191. Dupuy décrit à cette page la boucle de rétroaction caractéristique du temps du projet. Un point sur la gauche représente le passé, un point sur la droite l'avenir; une flèche reliant le passé à l'avenir indique que le passé produit causalement le futur, en même temps qu'une flèche reliant symétriquement l'avenir au passé indique que l'anticipation du futur provoque une réaction sur le passé. Cela fait-il sens? Car enfin, il s'agit du présent, non du passé! La boucle de rétroaction devrait agir sur notre action présente, non sur ce qui est déjà du passé pour nous! Pour le dire autrement: notre présent est le passé de notre avenir, et en anticipant l'avenir qu'on veut faire rétroagir sur le présent, ce n'est pas le passé que l'on modifie, mais notre présent et son avenir immédiat. Le présent est, devrait-on dire, le passé conditionnel d'un futur qui n'existe pas encore. C'est le présent (c'est-à-dire le passé conditionnel du futur) et le futur (et non le passé et le futur) qui se conditionnent réciproquement! 
près ${ }^{27}$, l'idée étant que «c'est parce que la dissuasion n'est qu'imparfaitement efficace qu'elle échappe à l'autoréfutation ${ }^{28}$. Sur ces points décisifs qui constituent la solution des apories successives pistées par Dupuy, celui-ci ne se contente hélas que de «quelques notations finales». On aimerait que soient notamment éclaircis les liens entre la psychologie de la motivation (puisque la lacune qu'il s'agissait de combler chez Jonas, c'était bien celle de la motivation à agir) et la logique, dont la force est précisément d'éliminer toute psychologie; mais garde-telle alors la force de motivation recherchée? D'autant que l'un des constituants du temps du projet semble être l'imagination de l'avenir, la capacité de se le représenter (l'image de l'avenir doit être à la fois repoussante et crédible) - autant de concepts psychologiques.

Mais quoi qu'il en soit de ces difficultés théoriques, l'allégeance de Dupuy à la pensée catastrophiste ne fait pas de doute, et ce par deux traits majeurs: d'une part - trait qu'il partage bien sûr avec Jonas -, le fait d'ordonner le raisonnement pratique en général, et la précaution en particulier, à la représentation de la catastrophe possible, point de focalisation de sa réflexion; à ce titre, sa pensée est bel et bien une pensée de la catastrophe, délibérément hyperbolique - une pensée salvatrice peut-être eu égard aux dangers qui nous menacent, mais dont il faut dire aussi qu'elle n'est guère transposable à la précaution plus modeste de nos laboratoires de recherche. Il faut donc la prendre pour ce qu'elle est, une contribution à la pensée sotériologique dont il faut reconnaître que même sous l'angle de la précaution, elle reste à peu près muette sur les pratiques quotidiennes de bricolage techno-scientifique auxquelles la précaution doit aussi s'appliquer. D'autre part, sa pensée est catastrophiste au sens rigoureux où elle cherche à établir une rationalité de la catastrophe ou de la pensée catastrophiste, s'opposant frontalement à tous ceux pour qui la pensée de la catastrophe est par définition irrationnelle, entraînant une paralysie de la réflexion. Dupuy prouve au contraire qu'il peut y avoir, qu'il doit y avoir une logique de la catastrophe.

\section{b. L'école prudentielle}

A l'opposé de la pensée catastrophiste, et pour cela même radicale, de la précaution, l'école prudentielle est bien plutôt taillée à la mesure du ventre mou du risque quotidien. Rien n'illustre mieux l'école prudentielle de la précaution que ce texte de celui qui en est le chantre, Philippe Kourilsky: «Au total, ce n'est pas un jeu de mots d'affirmer que le principe de précaution doit gouverner la mise en œuvre de la précaution. Cette apparente tautologie traduit le fait que, tout comme la prévention, la précaution est fille de la prudence. Celle-ci s'impose aux acteurs publics et privés, dès lors que leurs décisions présentent des risques potentiels ou avérés. La prudence implique de réfléchir à la portée et aux conséquences de ses actes et de prendre des dispositions pour éviter de causer des dommages à autrui. Inscrit dans le cadre de la prudence, le principe de précaution exprime la demande sociale d'une diminution des risques (...) Les convergences entre précaution, prévention et prudence pourraient justifier que l'on remplaçât le prin-

27 PCE 214.

28 PCE 213. 
cipe de précaution par un principe de prudence qui engloberait précaution et prévention $»^{29}$.

Malgré son unité nominale avec une notion bien connue dans la philosophie classique, la prudence, même définie de façon si englobante, n'a que peu à voir avec la prudence ou phronesis telle qu'Aristote en a élaboré le concept. Pour Aristote et ses héritiers, la prudence est avant tout une vertu, et une vertu intellectuelle, c'est-à-dire une certaine disposition à agir accompagnée, dit-il, d'une règle vraie $^{30}$; la prudence est donc la vertu qui dispose au choix d'un critère (ou règle; c'est pourquoi c'est une vertu intellectuelle) d'action (c'est pourquoi c'est une disposition pratique) dans le contexte d'un savoir portant sur le contingent (non pas sur ce qui est bien ou mal en soi, mais sur ce qui est bien ou mal pour l'homme). Ce qui entre autres choses distingue foncièrement cette prudence classique de la prudence moderne, c'est que la prudence aristotélicienne est étrangère à toute notion de calcul des conséquences; elle est avant tout une vertu qui s'incarne dans le personnage du phronimos qui pour cette raison même a valeur d'exemple pour la communauté. Agir bien ou mal dans des circonstances données, c'est pour le phronimos agir non en fonction de conséquences calculables, mais en fonction de sa connaissance de la nature humaine à laquelle il a, en tant que phronimos précisément, un accès privilégié. Son savoir n'est pas théorique au sens de la science du général, mais pratique, au sens d'une connaissance indissolublement liée à l'ici et au maintenant. La bonté de l'acte ne dépend en rien de ses conséquences, mais de son adéquation, dans sa singularité présente, à ce que le phronimos sait être valeureux.

On voit ainsi par contraste que le paradigme de la prudence moderne est tout entier gouverné par le modèle scientiste du calcul des conséquences. Il est donc quelque peu trompeur de faire de la précaution la fille de la prudence; il serait plus rigoureux d'en faire le dernier rejeton du conséquencialisme, et la cousine germaine de la théorie du choix rationnel - deux filiations qu'illustre au demeurant la version catastrophiste de la précaution. Mais quoi qu'il en soit de ces problèmes de pedigree, on voit que situer ainsi le principe de précaution tout entier dans le champ de la prudence ne fait guère que brouiller les cartes conceptuelles: car dans cette injonction de prudence généralisée, précaution, prévention et prudence sont rendues équivalentes; et si la précaution-prudence «implique de réfléchir à la portée et aux conséquences de ses actes et de prendre ses dispositions pour éviter de causer des dommages à autrui», alors elle n'est qu'une version molle et abâtardie de ce que n'importe quelle éthique libérale baptise depuis Max Weber du nom d' «éthique de la responsabilité». Point n'est besoin pour cela de mobiliser la batterie conceptuelle de la précaution.

\section{c. L'école dialogique}

«Démocratiser la démocratie»: tel pourrait être le mot d'ordre de l'école discursive ou dialogique de la précaution, qui utilise celle-ci comme levier d'une revivification participative d'une démocratie sclérosée dans des structures trop délégatives ou représentatives. Tel est le sens du plaidoyer de Michel Callon,

Philippe Kourilsky, Du bon usage du principe de précaution, Paris, Odile Jacob, 2002, pp. 48-49.

3o Voir à ce propos le livre classique de Pierre Aubenque, La prudence chez Aristote, Paris, P.U.F., 1993 (1 $1^{\text {ere }}$ éd. 1963). 
Pierre Lascoumes et Yannick Barthe dans leur livre déjà cité Agir dans un monde incertain. Le PP devient alors le paradigme d'un nouveau modèle de la décision démocratique, non plus une décision «tranchée et tranchante», mais plutôt une décision par «apprentissages collectifs, qui produisent simultanément de nouveaux savoirs et de nouvelles configurations sociales, [et] finissent par fabriquer une trame serrée de microdécisions, chacune d'entre elles étant soumise à discussion, liée à celles qui la précèdent mais également à celles qui la suivront $\gg^{31}$. Le PP est donc avant tout analysé comme un outil procédural permettant de dépasser les habituels modes de consultation d'experts et, plus généralement, de rendre aux acteurs sociaux la maîtrise de leur destin. Ce sont les «forums hybrides» qui sont le lieu privilégié de cette démocratie régénérée: «Forums, parce qu'il s'agit d'espaces ouverts où des groupes peuvent se mobiliser pour débattre de choix techniques qui engagent le collectif. Hybrides, parce que ces groupes engagés et les porte-parole qui prétendent les représenter sont hétérogènes: on y trouve à la fois des experts, des hommes politiques, des techniciens et des profanes qui s'estiment concernés. Hybrides, également, parce que les questions abordées et les questions soulevées s'inscrivent dans des registres variés qui vont de l'éthique à l'économie en passant par la physiologie, la physique atomique et l'électromagnétisme $»^{32}$.

L'hypothèse de fond qui guide les auteurs est celle de la fécondité de la controverse, facteur d'enrichissement de la démocratie; les forums hybrides en sont l'instrument adéquat, eux qui, débordant les savoirs d'expert bien cadrés, permettent d'explorer des mondes possibles qui ébranlent les certitudes instituées. Surgissent alors de nouveaux scénarios possibles, mais aussi de nouveaux acteurs sociaux qui jusque là n'avaient pas accès à la parole, ainsi que de nouvelles solutions à ce jour inenvisagées: c'est ce qui fait de la controverse ainsi organisée en forums hybrides un processus d'exploration et d'apprentissage scientifiques. En sociologue des sciences qu'il est, Michel Callon identifie trois points d'entrée possibles des profanes dans le cycle de production de la science, déconfinant ainsi la science confinée: $1^{\circ}$ au niveau de l'accumulation primitive des connaissances, lorsque des «groupes concernés » s'acharnent à rendre visibles des phénomènes inexpliqués qui les atteignent; $2^{\circ}$ en entrant dans le «collectif de recherche», s'immisçant, forts de leur point de vue de profane, dans des controverses scientifiques en cours; $3^{\circ}$ en faisant valoir leur savoir pratique de profanes face aux connaissances confinées dans l'espace du laboratoire - trois points d'entrée qui ne visent pas tant à disqualifier la science de laboratoire qu'à étayer un plaidoyer pour une nouvelle coopération entre la science confinée et «la recherche de plein air».

Plus fondamentalement, le modèle des forums hybrides doit donc conduire à redistribuer les cartes de l'espace communicationnel de nos démocraties, en abolissant la double coupure qui sépare l'expert du profane et les citoyens de ses représentants (les auteurs parlent aussi à ce propos de double monopole) pour instaurer des procédures authentiquement dialogiques (à l'opposé, par exemple, du sondage d'opinion, dont il est noté avec pertinence qu'il «renforce les méca-

AMI 26.

32 AMI 36. 
nismes par lesquels la démocratie délégative se protège contre la démocratie dialogique $»^{33}$; une analyse similaire est faite à propos du référendum). C'est par le biais de ce plaidoyer pour la nécessaire intégration de procédures dialogiques devant aboutir non à l'inaction, mais à l'action mesurée, que les auteurs retrouvent le PP; pour les auteurs en effet (mais il s'agit de toute évidence de Pierre Lascoumes), «la précaution désigne une démarche active et ouverte, contingente et révisable. Elle est exactement l'inverse d'une décision tranchée une fois pour toutes $»^{34}$.

A l'inscrire ainsi dans le cadre d'une critique générale de nos usages démocratiques, le PP devient donc l'équivalent fonctionnel de la décision «tranchante et tranchée» propre à la démocratie représentative, qu'elle soit faite par des experts, par un gouvernement ou par un vote référendaire. Mais tout en assumant cette fonction décisionnelle, le PP fait subir à la décision un double changement, à la fois dans la manière dont elle est prise - puisqu'elle subvertit la traditionnelle division du savoir entre le savoir d'expert et le savoir commun - et dans sa nature même - puisque, à l'inverse de la décision salomonesque, elle reste une décision ouverte, révisable, sensible à l'émergence de savoirs nouveaux. Dans cette version, le PP apparaît donc comme un mode décision, à la fois procédural et communicationnel: procédural, parce qu'il ne préjuge en rien de la décision substantielle à prendre, se contentant de garantir les conditions équitables d'un échange de points de vue et d'informations aussi complet que possible (c'est l'idée des forums hybrides); communicationnel, parce que la décision n'est plus celle d'un seul qui décide souverainement après consultation, mais procède au contraire d'un processus de mise en commun par les acteurs sociaux concernés de réinterprétations successives de situations d'incertitude à la lumière de connaissances et d'informations évolutives.

Mettre les forums hybrides au service d'une démocratisation de la démocratie, c'est une chose qui se comprend fort bien. On s'étonne d'ailleurs que les auteurs ne citent pas une seule fois Habermas, chez lequel ils auraient sans doute trouvé l'armature théorique la plus appropriée à leur propre projet ${ }^{35}$. Mais faire du principe de précaution - que précautionneusement ils baptisent le plus souvent de «la précaution», sans doute pour lui donner subliminalement une extension plus grande - un principe de démocratie en est une tout autre, qui ne laisse pas de surprendre. Qu'un bon usage du principe de précaution soit un usage démocratique, cela n'est pas douteux; mais cela ne fait pas de la précaution un principe démocratique pour autant, de la même manière que la Passion du Christ n'est pas un événement musical du simple fait que c'est la musique qui en a donné l'expression la plus haute. C'est confondre le champ d'application de la chose avec sa nature: si le principe de précaution devait prioritairement s'appliquer à la sélection des futurs curés parmi les jeunes séminaristes, cela n'en ferait pas ipso facto un principe de politique ecclésiale. Autrement dit: il est vrai que pour des raisons qui tiennent à la nature du PP - et sur lesquelles nous reviendrons encore -, celui-

\footnotetext{
AMI 213.

AMI 264.

Voir par exemple son Droit et démocratie, trad. de Rainer Rochlitz et Christian Bouchindhomme, Paris, Gallimard, 1997, ainsi que son article, extrêmement éclairant à cet égard, «La souveraineté populaire comme procédure», trad. Mark Hunyadi, in: Lignes, octobre 1989.
} 
ci est particulièrement approprié à un usage démocratique: puisqu'il s'agit souvent de faire valoir des hypothèses déviantes par rapport à l'orthodoxie scientifique, industrielle ou politique, puisque les dommages éventuels concernent ce qu'on appelle des groupes «latents», c'est-à-dire non constitués en tant que groupe et à ce titre sans accès à la représentation, puisqu'il est presque impossible de déterminer à priori quels sont ou seront les acteurs sociaux concernés, la mise en place de structures communicationnelles et démocratiques inédites est effectivement un outil en tout point recommandable pour une application optimale du PP. Mais le PP existerait même sans démocratie pour le mettre en œuvre, et il ne lui est pas plus lié que le Code civil au papier sur lequel il est imprimé. Cela apparaît donc comme une confusion supplémentaire que d'identifier purement et simplement le principe de précaution avec le principe de démocratie, comme le font les tenants de l'école «dialogique» de la précaution.

\section{L'APORIE DE LA PRÉCAUTION}

Le problème conceptuel général qui se pose à propos du PP est de le distinguer d'un simple principe de prévention, avec lequel on le confond si souvent. Si l'on n'arrivait pas à établir cette distinction, alors il apparaîtrait clairement que l'invocation tous azimuts du PP auquel on assiste depuis le début des années 1990 est en réalité purement rhétorique et ne sert qu'à auréoler d'un nouveau nom un principe d'action qui n'a jamais été étranger aux politiques publiques; la précaution ne serait alors que l'habit pailleté dont on revêt la bonne vieille prévention.

La première partie de la thèse ici défendue consiste à dire que l'on s'interdit de penser réellement la distinction entre précaution et prévention, si l'on s'en tient pour ce faire à une distinction opérée en fonction de la nature des risques. C'est pourtant ce que l'on fait le plus souvent, comme en atteste une large partie de la littérature consacrée à ce sujet, et encore dans cette nouvelle bible du risque qu'est le Traité des nouveaux risques de Godard, Henry, Lagadec et Michel-Kerjan ${ }^{36}$. On peut en effet y lire - mais c'est un leitmotiv de la littérature sur le sujet - que «lorsque les phénomènes qui sous-tendent le danger sont scientifiquement bien compris et que leur probabilité est connue, on a affaire à des risques avérés dont l'existence ne fait pas de doute et dont seule la réalisation est aléatoire. Ces risques classiques (accidents de la route, tempêtes, inondations, etc.) relèvent de démarches de prévention. (...) La répétition de phénomènes de même nature permet la construction de bases de données statistiques sur la longue durée, moyennant un investissement dans le recueil systématique des informations. (...). Rien de cela n'est acquis en l'absence de certitude sur les phénomènes de base et sur l'existence du danger. Le risque y est hypothétique, soupçonné. Il est fait d'interrogations, de présomptions plus ou moins solidement étayées, de scénarios plus ou moins plausibles. Un risque hypothétique, non avéré, ne peut cependant pas être considéré comme un risque très peu probable, voire négligeable. C'est une possibilité identifiée de risque dont on ne connaît précisément pas la probabilité ${ }^{37}$. C'est en ce cas la précaution qui s'applique, alors que le principe de

36 cf. note 3 . 
prévention s'applique aux seuls risques avérés. Les deux principes sont à chaque fois corrélés à des risques de nature différente: c'est la nature du risque qui décide du principe à appliquer.

La même idée est réaffirmée avec force par Pierre Lascoumes: «Un risque potentiel est construit à partir d'un faisceau d'indices et d'hypothèses qui ne sont pas encore scientifiquement validées mais permettent de déclencher une alerte. Son identification repose sur la mise en relation d'informations hétérogènes, produites aussi bien par la recherche confinée que par la recherche de plein air, qui permettent progressivement de cantonner l'incertitude. Experts et profanes disposent chacun de savoirs complémentaires qui permettent d'avancer dans l'identification du danger, dans les façons de le cerner et d'organiser une démarche de précaution limitant d'éventuels dommages. Une fois le risque avéré, c'est-à-dire connu dans ses manifestations et expliqué, les décisions ultérieures relèvent de la prévention ${ }^{38}$. A dire vrai, peu de textes rassemblent de manière aussi dense ce qu'on peut à bon droit appeler l'aporie de la précaution. Le risque potentiel est subrepticement assimilé à un danger, dont l'identification doit entraîner une démarche de précaution dont le but est de limiter d'éventuels dommages. Cela signifie, à n'en pas douter, que des mesures ont été prises afin d'éviter certains risques potentiels; mais ces risques potentiels, il a bien fallu les considérer comme des dangers réels (d'où le glissement, dans le texte, du «risque potentiel» au «danger»), sans quoi on n'aurait pas pris de mesures pour les éviter. Mais où est, alors, la différence avec la prévention? On dira: dans la certitude du risque. Mais cette différence s'évanouit au moment même où on la formule: car dans les deux cas, risques hypothétiques et risques avérés, le but de l'action envisagée est à chaque fois d'éviter un certain dommage. Les mesures prises visent ici comme là à éviter que n'advienne une conséquence indésirable. Mais puisque la visée commune est l'évitement d'un dommage, et puisque pour l'éviter, il faut prendre des mesures, cela ne fait aucune différence que le risque envisagé soit hypothétique ou avéré; si l'on veut vraiment éviter la réalisation de certains risques, alors on est obligé de traiter les risques hypothétiques comme des risques avérés: sans quoi, encore une fois, on ne prendrait pas de mesures pour les éviter. Mais si l'on est contraint de considérer les risques hypothétiques comme des risques avérés, alors il ne sert plus à rien de vouloir les distinguer, et ce n'est certainement pas sur une distinction aussi inopérante dans les faits que l'on pourra à son tour fonder la différence entre principe de précaution et principe de prévention. La distinction conceptuelle s'évanouit sous l'urgence impérieuse d'éviter des conséquences jugées par trop dommageables; c'est l'aporie de la précaution.

De cette assimilation qui rend vaine la distinction qu'elle cherche pourtant à fonder, on a la preuve éclatante chez les mêmes auteurs qui déclarent un peu plus loin, sans sourciller devant la contradiction aveuglante qui se signale comme un néon rouge à chaque étape de leur raisonnement: "On trouve en particulier dans la communication de la commission ${ }^{39}$ la recommandation de veiller à ce que les

TNR, pp. 125-126.

AMI 271. 
mesures prises au nom de la précaution pour des risques hypothétiques soient comparables par l'ampleur et la nature à celles adoptées pour des risques avérés similaires $\gg^{40}$. Comment avouer plus clairement que la distinction prétendument fondatrice entre risques hypothétiques et risques avérés est de ce point de vue-là tout simplement nulle et non avenue, et qu'elle ne peut en conséquence rien fonder du tout? Il faut en effet, selon les auteurs, comparer les mesures prises face aux risques hypothétiques (précaution) aux mesures adoptées pour des risques avérés similaires (prévention): mais qu'est-ce que cela peut bien vouloir dire d'autre que de recommander de traiter les risques hypothétiques comme des risques avérés? En outre, comment un risque avéré peut-il être déclaré similaire à un risque hypothétique, que par définition l'on ne connaît pas? Qu'a exactement d'hypothétique un risque similaire à un risque avéré? L'aveu est complet: l'exigence d'évitement des risques abolit totalement, de fait, la distinction entre risques hypothétiques et risques avérés, en obligeant dans les deux cas à traiter les hypothèses comme des certitudes.

Telle est la première raison pour laquelle il est foncièrement erroné de fonder la distinction entre principe de précaution et principe de prévention en fonction de la nature des risques envisagés. Mais il en est une deuxième, qui va nous mettre plus directement sur la voie de la résolution de notre problème de démarcation. Nous venons de voir la difficulté conceptuelle qu'il y avait à distinguer précaution et prévention en fonction de la nature des risques encourus, hypothétiques ou avérés. Une autre raison permet de la mettre en évidence. Si l'on admet en effet que cette visée commune de l'évitement oblige en réalité à abolir la distinction entre risques hypothétiques et risques avérés - et cette conclusion est inévitable, dès lors qu'il s'agit de mesures à prendre -, alors cette prétendue distinction se heurte à la conclusion paradoxale suivante: puisque, selon ce qui vient d'être dit, les risques hypothétiques devraient faire l'objet de mesures d'évitement aussi contraignantes que les risques avérés - parce que le fait même de prendre des mesures d'évitement implique qu'on considère la réalisation du risque comme causalement établie -, et ce, indépendamment du degré de certitude qui lui est associé, la simple possibilité d'un risque maximal devrait nécessairement entraîner la mesure d'évitement correspondante, quel que soit son degré de plausibilité. Et comme par définition, le champ des risques possibles est incommensurablement plus grand que celui des risques avérés, on devrait prendre incommensurablement plus de mesures d'évitement pour les risques possibles que pour les risques avérés. On ne saurait caractériser plus précisément la paralysie totale de l'action.

\section{LA LOGIQUE DU RAISONNEMENT DE PRÉCAUTION}

Référence est faite à la communication de la Commission européenne (2000) qui recommande que «les mesures fondées sur le principe de précaution devraient:

être proportionnées au niveau de protection recherché (...)

être cohérentes avec des mesures similaires déjà adoptées (les mesures devraient être d'une portée et d'une nature comparables à celles des mesures déjà prises dans des domaines équivalents o ?u toutes les données scientifiques sont disponibles) (...)». Cf. TNR, p. 121.

40 TNR, p. 130. 
Cette conclusion est évidemment absurde, et assurément aucun des auteurs cités ne la ferait sienne. Elle est pourtant nécessaire, et découle de leur amorce de départ, qui veut distinguer précaution et prévention sur la seule base de la nature des risques. Ce que montre notre raisonnement par l'absurde, c'est que cette base est erronée: on ne peut logiquement fonder la distinction entre précaution et prévention sur la base de la seule distinction entre risque hypothétique et risque avéré. Pour le dire autrement: ce qui compte, ce n'est pas la nature des risques en général, mais la nature des hypothèses qui font d'un risque un risque hypothétique. La distinction matérielle entre risques hypothétiques et risques avérés que font ces auteurs - elle est matérielle au sens où elle ne peut reposer que sur des bases strictement empiriques - efface au profit d'une seule caractéristique (pour le risque, d'être hypothétique ou avéré) la vaste palette des distinctions logiques possibles au sein même des hypothèses susceptibles de faire d'un risque un risque hypothétique (ces distinctions sont dites logiques au sens où Popper parle de la «logique du raisonnement scientifique», qui implique donc l'examen du type, de la nature - déterminée par exemple par la testabilité différenciée - des hypothèses en présence). Or, c'est cette palette et cette palette seule qui importe pour l'application du principe de précaution. Le principe de précaution ne s'applique pas aux risques hypothétiques du simple fait que ce sont des risques hypothétiques, il s'applique à toutes les situations d'incertitude qui pour établir cette incertitude font appel à un raisonnement hypothétique. Telle est la logique du raisonnement de la précaution. La nuance peut paraître faible par rapport à la position que nous avons critiquée, elle marque en réalité toute la différence qui sépare une détermination matérielle du principe de précaution d'une détermination logique; ce qui lui donne à la fois l'avantage de la rigueur conceptuelle et de la validité pratique.

En effet, cette manière logique de caractériser le principe de précaution permet d'échapper au premier paradoxe conceptuel dans lequel s'enfermait la position classique, celui de devoir traiter en définitive les risques hypothétiques comme des risques avérés, alors même que c'est la différence entre les deux qu'elle voulait établir. Il n'y a en effet plus de paradoxe à dire que l'on veut éviter, au même titre que les risques avérés, des risques hypothétiques, dès lors que les hypothèses qui les établissent sont suffisamment étayées; alors qu'il y en a un à vouloir fonder une distinction que sa mise en œuvre abolit. La nuance n'est pas seulement dans la manière de dire - encore qu'en matière conceptuelle, la manière de dire soit bien évidemment l'essentiel. Les partisans de la distinction classique, une fois qu'ils ont établis la distinction précaution / prévention sur la base de la nature des risques, doivent ensuite formuler une quantité de distinctions d'appoint (notamment quant à la plausibilité des risques), sans doute pour éviter les paradoxes auxquels ils seraient acculés s'ils n'en formulaient point: car ils doivent bien justifier que tout risque hypothétique ne justifie pas per se une mesure de précaution, ce qu'ils ne peuvent faire qu'en distinguant les risques hypothétiques plausibles de ceux qui ne le sont pas; au lieu qu'en nous concentrant d'emblée sur la nature des hypothèses mises en œuvre par le raisonnement de précaution, nous mettons immédiatement en évidence la base logique du principe de précaution, sans devoir multiplier les distinctions matérielles ayant trait aux types de risques et situations envisageables. Nous y gagnons en économie, donc en clarté. 
Mais du coup s'évanouit aussi le deuxième paradoxe, celui qui conduisait finalement à devoir éviter un nombre incommensurable de risques hypothétiques, quel que soit leur degré de plausibilité. Cette conséquence paradoxale (et manifestement intenable) est évitée du fait que notre formulation du principe de précaution met dès l'abord l'accent, comme nous venons de le voir, sur la nature des hypothèses impliquées dans les situations d'incertitude. Il en résulte immédiatement que ce n'est pas tant la nature hypothétique du risque qui sollicite le recours au principe de précaution que la nature des hypothèses qui font que le risque en question soit un risque hypothétique. Il est ainsi d'emblée entendu que l'identification de conséquences indésirables peut reposer sur des hypothèses plus ou moins plausibles, plus ou moins observables, plus ou moins testables et critiquables; que toutes les hypothèses ne se valent évidemment pas, et qu'il ne suffit pas qu'un risque soit hypothétique pour qu'il faille devoir l'éviter à tout prix. Si l'on se décide toutefois à l'éviter par des mesures que l'on estime adéquates, c'est parce que son identification repose sur des hypothèses suffisamment étayées. Encore une fois, ce n'est pas la nature du risque qui nous enjoint à prendre des mesures de précaution, mais la nature des hypothèses qui en établissent la réalité.

La rigueur conceptuelle nous oblige donc à établir la différence entre principe de précaution et principe de prévention non sur la base matérielle de la différence des risques encourus, mais sur la base logique du type de raisonnement à chaque fois mis en œuvre. Il s'agit donc ultimement d'une question de logique de la justification: comment justifie-t-on des mesures d'évitement? Si la justification fait apparaître des hypothèses, alors les conclusions ressortissent à la précaution; sans ce recours à des hypothèses, les conclusions relèvent de la prévention. Dans les deux cas, il est vrai, la conclusion (matérielle) est la même: des mesures d'évitement jugées adéquates. Mais formellement, la nature de cette conclusion sera différente selon le type de raisonnement qui y a conduit: dans le cas d'un raisonnement qui ne fait intervenir d'hypothèses à aucune étape de son déroulement, la conclusion d'évitement sera dite résulter du principe de prévention; à l'inverse, dans le cas d'un raisonnement qui fait intervenir une hypothèse à l'une quelconque de ses étapes, la conclusion sera dite résulter du principe de précaution. L'identité de la conclusion matérielle ne doit pas masquer la différence du type de raisonnement qui y conduit, et c'est cette différence logique qui fonde la différence entre précaution et prévention.

Il ne s'agit toutefois donc pas pour nous de nier la différence entre risques hypothétiques et risques avérés, mais de dire que c'est là une distinction empirique sur laquelle on ne peut pas fonder la logique - soit de précaution, soit de prévention - qui prévaut lors de la mise en œuvre de mesures d'évitement; car ce qui compte dans le raisonnement de précaution, ce n'est pas la nature des risques, mais la valeur des hypothèses. On dira: mais la valeur d'une hypothèse ne peut elle aussi se décider, en dernière analyse, qu'empiriquement, et selon les standards scientifiques et de rationalité disponibles: à la distinction entre les risques, vous n'avez fait que substituer une distinction entre les hypothèses. Le gain semble peu évident, d'autant que finalement, malgré le déplacement que vous effectuez de la définition matérielle des risques à la logique du raisonnement de précaution, le résultat reste le même: pour vous comme pour la définition classique, le principe de précaution ne s'applique en définitive qu'à ce que vous aussi appelez les risques hypothétiques! La position classique part de la définition des risques 
hypothétiques, vous, vous parlez d'hypothèses qui interviennent dans le raisonnement, mais l'aboutissement est le même: c'est tout de même à chaque fois d'hypothèses dont on parle. Votre passage à la logique du raisonnement n'a donc aucun intérêt du point de vue pratique...

On répondra: même si cette discussion manque peut-être d'intérêt immédiat pour le praticien de la précaution, trois avantages sur la théorie classique se sont néanmoins déjà dégagés de notre approche: $1^{\circ}$ elle permet de rendre compte de la raison pour laquelle tous les risques hypothétiques ne justifient pas par euxmêmes l'adoption de mesures de précaution, ce que la théorie classique ne peut pas faire sans opérer des distinctions d'appoint; $2^{\circ}$ elle permet d'échapper aux deux paradoxes dans lesquels s'enferre inévitablement la théorie classique; $3^{\circ}$ enfin, elle met en évidence la nature même de la précaution, en en faisant non un principe d'action - ce qui rend impossible la distinction avec la prévention - mais une manière de raisonner: on a passé de la description de l'action précautionneuse à la logique du raisonnement de précaution. C'est ce dernier point dont la pertinence est contestée par notre contradicteur, puisque sous couvert de rechercher la logique de la précaution, on aurait en fait remplacé une distinction empirique par une autre. Mais tel n'est évidemment pas le cas. S'intéresser à la base empirique d'une hypothèse, à sa testabilité ou falsifiabilité, sa pertinence ou son observabilité empiriques, n'implique pas du tout que le raisonnement qui fait appel à ces hypothèses soit lui-même empirique, tout aussi peu que la règle $« 2+2=4$ » ne devient pas empirique du simple fait que je peux l'appliquer à des pommes. Le raisonnement de précaution est un raisonnement pratique, au sens où il débouche inévitablement sur une règle d'action (qui peut bien entendu être une règle d'inaction ou d'abstention), mais en tant que raisonnement pratique, il a ses règles; et ce n'est certainement pas parce que ces règles font intervenir des hypothèses empiriques que le raisonnement lui-même devient empirique. Ce qui importe pour la logique du raisonnement, c'est le fait même qu'il s'agisse d'hypothèses, non la qualité intrinsèque de ces hypothèses; c'est à la pratique du raisonnement qu'il reviendra d'éprouver la qualité des hypothèses à la lumière des standards de rationalité disponibles. Qu'un raisonnement fasse intervenir une mauvaise hypothèse ne le rend pas moins hypothétique pour autant.

Le raisonnement hypothétique a donc son armature logique propre, et la nature empirique des hypothèses ne l'infirme en aucune manière. En revanche, l'intervention d'hypothèses rend assurément le raisonnement pratique fort complexe. La prémisse de départ est une action envisagée - la culture d'OGM en plein champ, l'implantation d'une nouvelle centrale nucléaire, la commercialisation d'un nouveau médicament - et la conclusion une règle d'action permettant de décider de l'opportunité de l'action envisagée. Entre la prémisse et la conclusion intervient une chaîne complexe où il faut $1^{\circ}$ identifier des hypothèses quant aux événements susceptibles de survenir à la suite de l'action envisagée; $2^{\circ}$ évaluer leur plausibilité; $3^{\circ}$ identifier leurs conséquences possibles; $4^{\circ}$ identifier leur plausibilité; $5^{\circ}$ évaluer leur désidérabilité. La conjonction linéaire de ces cinq étapes donne la base, $6^{\circ}$, de l'appréciation permettant d'établir, et $7^{\circ}$, la règle d'action correspondante, qui constitue la conclusion du raisonnement de la précaution. Convertir cette règle d'action en décision n'appartient plus au raisonnement luimême, qui se limite à justifier la règle d'action qui à son tour motivera la décision. On peut visualiser cela selon le schéma de la figure suivante: 


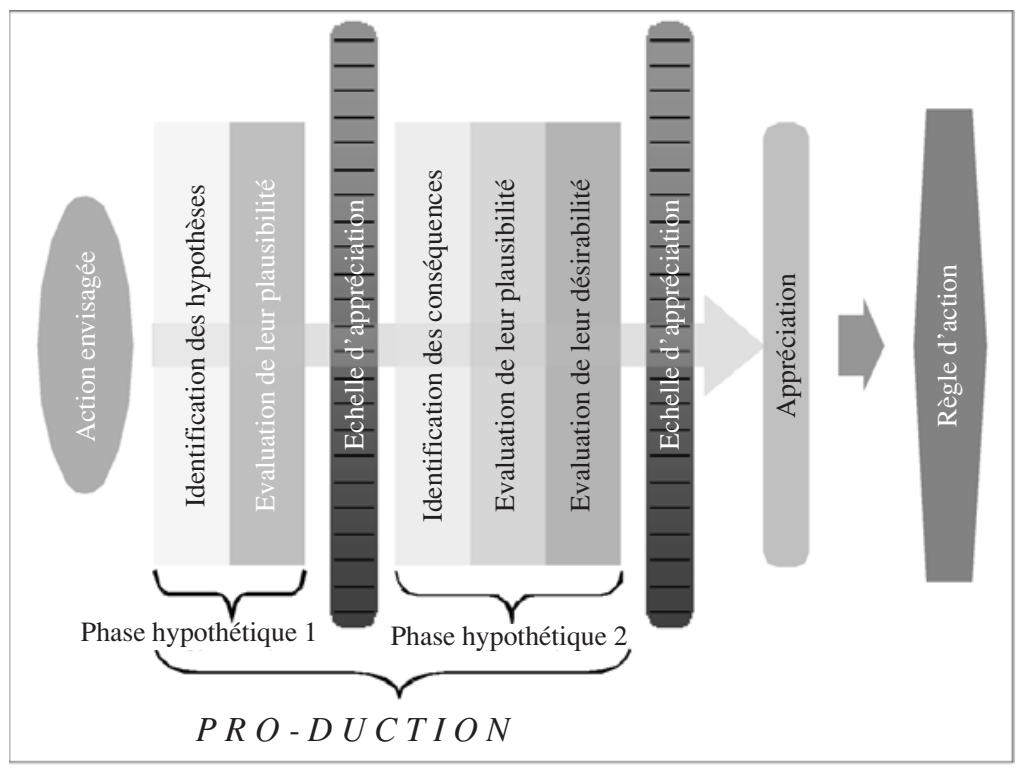

On voit s'y succéder deux phases hypothétiques ( $1 \& 2)$, dont la première concerne les hypothèses elles-mêmes (ce sont des hypothèses sur les hypothèses: quels événements sont susceptibles d'arriver?), et la deuxième leurs conséquences (hypothèses sur les conséquences: quelles conséquences les événements susceptibles d'arriver peuvent-ils entraîner?). Chacune de ces phases se subdivise à son tour une étape descriptive et une étape évaluative. En effet, une fois établie la liste des événements susceptibles d'arriver, il faut évaluer leur plausibilité; et une fois établie la liste des conséquences possibles, il faut évaluer à la fois leur plausibilité et leur désidérabilité.

L'action envisagée est la suivante ${ }^{41}$ : créer par transfert de gène une plante résistant aux nématodes, un ver microscopique qui vit dans tous les milieux naturels. Plusieurs espèces de ce petit ver s'attaquent en particulier aux plantes cultivées, provoquant fréquemment de graves dommages notamment au niveau des racines. Il n'est donc pas inutile de noter que cette voie de recherche entend résoudre un problème agronomique réel, et qu'il ne s'agit pas simplement d'une amélioration visant des fins de simple augmentation de rentabilité: ce sera un aspect important lorsque, au terme du raisonnement de précaution, l'on devra envisager une règle d'action (à l'étape $7^{\circ}$ ). Actuellement, les seuls pesticides capables de lutter efficacement contre les nématodes sont si toxiques tant pour l'environnement, les animaux et l'homme que leur usage est interdit; et la seule alternative aujourd'hui disponible à ces poisons est la rotation des cultures. Mais

${ }^{41}$ Je dois cet exemple à Pia Malnoe, chercheuse à la station fédérale de recherche en production végétale à Changins (Suisse) et membre du groupe RIBios (Genève). Je la remercie pour sa patiente collaboration. 
dans certaines situations, elle est quasiment impossible, comme dans le cas des vignes par exemple. La solution envisagée consiste donc à introduire dans la plante que mange le nématode une séquence d'ADN qui code pour une ARN double brin qui va inactiver un gêne vital du nématode - impliqué par exemple dans la formation de l'embryon. On a en effet observé qu'un double brin d'ARN (qui dans ce cas ne code même pas pour une protéine) était capable d' inhiber l'expression d'un gène du nématode pour autant que la séquence d'ARN double brin corresponde exactement à la séquence du gène qu'on veut rendre silencieux du nématode. L'originalité de la méthode envisagée consiste en l'occurrence à inactiver un gène par l'intermédiaire de la nourriture ingurgitée, des racines de la plante par exemple.

L'action envisagée étant ainsi justifiée, doivent maintenant être identifiées les hypothèses générales concernant le type d'événements susceptibles de survenir lors d'une application plein champ $\left(1^{\circ}\right)$, étant entendu que la faisabilité technique et l'efficacité de cette méthode a été préalablement testée en laboratoire. Ce qui veut dire notamment que toutes les hypothèses qui seront identifiées en $1^{\circ}$ seront elles-mêmes placées sous l'hypothèse générale d'un fonctionnement biologique identique dans des conditions extérieures, par définition variables; des changements inattendus peuvent donc survenir une fois que la plante est transférée dans son écosystème naturel. Cette réserve générale étant faite, quatre hypothèses peuvent être, grosso modo, envisagées: 1.- celle d'une influence possible sur la plante elle-même; 2.- celle d'une influence sur le sol, en particulier sur les microorganismes qui y vivent; 3.- celle d'une influence possible sur les animaux, par ingestion; 4.- celle d'une influence possible sur l'homme, par ingestion également. Les hypothèses ainsi formulées sont nécessairement très générales: elles se spécifieront lors de l'identification des conséquences possibles qui leur sont liées. Elles reposent néanmoins sur un rapport de causalité de prime abord possible ou plausible. Il faut cependant noter que même à ce niveau très général, leur plausibilité $\left(2^{\circ}\right)$ peut, dans des proportions certes très variables, être évaluée en laboratoire. L'influence sur la plante, le sol, les animaux peut, dans une certaine mesure, être observée; l'influence sur l'homme doit être extrapolée à partir des observations faites sur les animaux, ce qui est à son tour une procédure problématique. Nous avons donc ici trois hypothèses à degré de plausibilité variable, et une à plausibilité inconnue. Si l'on passe à la troisième étape du raisonnement - l'identification des conséquences $\left(3^{\circ}\right)$-, alors on verra que cette hypothèse à plausibilité inconnue pourrait toutefois, si l'on envisage l'une des conséquences possibles qui pourraient se réaliser, s'avérer absolument indésirable (évaluation qui est l'objet de $5^{\circ}$ ): celle d'un dérèglement au niveau de la fertilité. Toutefois, la prise en compte de la plausibilité de cette conséquence $\left(4^{\circ}\right)$ nous montre que, toute indésirable qu'elle soit, cette conséquence a une probabilité extrêmement faible: pour qu'une séquence d'ARN puisse agir sur le gène correspondant, il faut que la séquence de 21 paires de bases qui compose l'ARN double brin soit absolument identiques à celles de l'ADN humain; or, si nous connaissons les bases composant le gène homologue chez l'homme, nous pouvons savoir par comparaison si l'ARN introduit dans la plante aura ou non un effet similaire chez l'homme. De plus, si nous connaissons l'ensemble du génome humain, nous pouvons savoir si cette séquence de 21 bases se retrouve ailleurs sur son $\mathrm{ADN}$, ce qui nous permet de savoir si l'ARN de la plante pourrait modifier un autre gène chez l'homme que 
celui de la production de gamètes. Si tel n'est pas le cas, alors il ne reste que le problème des variations individuelles: les 21 paires de bases homologues pourraient se retrouver chez tel ou tel individu. Cette probabilité est extrêmement faible, mais pas nulle. Ce raisonnement nous apprend toutefois qu'une modification génétique est, chez l'homme en tant qu' espèce, impossible; elle ne pourrait advenir que chez certains individus.

Nous avons suivi cette ligne de raisonnement pour une conséquence envisageable chez l'homme. Mentionnons à titre indicatif une conséquence à chaque fois possible dans chacun des autres cas considérés: en ce qui concerne l'influence sur la plante, il se pourrait que, désormais dotée d'un caractère qui l'avantage, elle devienne une mauvaise herbe; en ce qui concerne le sol, la méthode envisagée pourrait éventuellement changer l'équilibre des micro-organismes utiles au sol, et ainsi modifier l'écosystème de celui-ci, le rendant moins apte à la culture l'année suivante; quant aux animaux, comme pour l'homme, l'ingestion de ces plantes modifiées pourrait avoir une influence sur leur fécondité. Telles sont quelques conséquences identifiées $\left(3^{\circ}\right)$, et il y en a bien d'autres, qui toutes devraient être évaluées en termes de plausibilité $\left(4^{\circ}\right)$ et de désidérabilité $\left(5^{\circ}\right)$ avant de permettre la formation du jugement $\left(6^{\circ}\right)$. Impossible donc de traiter un tel exemple de manière exhaustive, tant se multiplient à chaque niveau les hypothèses envisageables et les manières de les évaluer. Le raisonnement de précaution est donc loin d'être linéaire, il se présente plutôt comme un processus itératif à embranchements multiples, aussi multiples que les hypothèses envisagées

La formation d'hypothèses scientifiques a traditionnellement été considérée sous le seul angle de la formation de conjectures explicatives, ce que l'on appelle depuis Charles Sanders Peirce l'abduction (ou rétroduction). Il s'agit donc dans ce type de raisonnement de remonter de faits observés à une conjecture explicative, donc d'aller du conséquent connu (le fait observé) à son antécédent inconnu (ce qui explique ce fait) à propos duquel on forme une hypothèse qui sera ensuite soumise à vérification, comme lorsque l'on cherche la cause des éclairs ou de l'agrandissement du trou de la couche d'ozone. De manière caractéristique, Peirce commence l'une de ses analyses consacrées à l'abduction en déclarant: "Toute recherche quelle qu'elle soit prend sa source dans l'observation (...) de quelque phénomène surprenant, de quelque expérience qui ou bien déçoit une attente ou bien rompt quelque habitude d'attente de l'inquisiturus $<\mathrm{du}$ chercheur $>{ }^{42}$. La surprise est à la source du questionnement du chercheur, et l'abduction est le nom de la démarche qui remonte d'un fait surprenant à une conjecture qui l'explique. Or, ce qui caractérise précisément nos situations d'incertitude, c'est que le mouvement va exactement en sens inverse: nous connaissons l'antécédent - l'action particulière que nous faisons ou envisageons de faire - mais pas le conséquent, c'est-à-dire ce qui résultera de cette action; nous formons donc des hypothèses non pas en amont de ce qui est connu, mais en aval, non pas à propos de ce qui précède ce que nous expérimentons mais à propos de ce qui le suit, non à propos du passé mais à propos du futur - c'est pourquoi on devrait ici parler, pour que la symétrie des mots reflète la symétrie des choses, non d'abduction ou de rétroduc-

42 Charles Sanders Peirce, «De la réalité de Dieu», texte paru dans Gérard Deledalle, Lire Peirce aujourd' hui, Bruxelles, De Boeck, 1990, p. 181. 
tion mais, en toute rigueur, de pro-duction, i.e. d'un mouvement de formation d'hypothèses orientées vers l'avant, le futur, l'incertain. Je propose donc de définir la pro-duction comme le raisonnement qui forme des hypothèses quant aux conséquents envisageables de phénomènes existants ou envisagés, et, par suite, de définir le principe de précaution comme la forme de raisonnement pratique qui met en ouvre la pro-duction.

L'abduction forme donc des hypothèses sur l'antécédent, là où la pro-duction le fait sur le conséquent. A la différence, cette fois, de l'induction qui, par vérification des hypothèses produites par abduction, conduit à la découverte de lois (Peirce dit aussi que «c'est la fonction spécifique de l'induction que de produire des propositions universelles et nécessaires $\left.{ }^{43} 》\right)$, la «pro-duction» ne vérifie rien, mais élabore des hypothèses sur la base du savoir disponible qui, lui, peut bien être inductivement fondé. Par exemple, dans le cas de l'élimination du nématode qui nous occupait à l'instant, c'est sur la base du savoir acquis en génétique que nous avons pu éliminer l'hypothèse élaborée par abduction d'une conséquence possible sur la fécondité humaine; et c'est aussi sur la base d'un savoir acquis que nous devons prendre au sérieux, entre autres hypothèses possibles, celle de la mauvaise herbe. Ces exemples montrent en outre que l'abduction - en tous cas dans un contexte de précaution - est prioritairement orientée vers des propositions singulières, alors que l'induction est par nature orientée vers des propositions universelles (une proposition universelle étant une proposition, comme le dit Peirce, «qui affirme quelque chose du tout d'un domaine ${ }^{44}{ }$, alors qu'une proposition singulière affirme quelque chose à propos de quelque chose de singulier). S'il y a des leçons générales à tirer d'une situation particulière (par exemple de l'élimination du nématode par une transgenèse effectuée sur la plante qui le nourrit), c'est à l'induction, et non à la pro-duction, qu'il reviendra de le faire. La production ne teste pas des hypothèses, mais lance des conjectures quant aux conséquences, comme l'abduction le fait quant aux causes.

Quoi qu'il en soit, cette définition de la précaution comme raisonnement pratique mettant en œuvre la pro-duction nous met très loin de la définition de la précaution comme vertu, puisqu'elle n'est pas tant une disposition qu'une manière de raisonner. Mais de la vertu aristotélicienne, elle garde tout de même son aspect intellectuel (dianoétique, dit-on dans les traductions d'Aristote) : car la finalité propre du raisonnement de précaution est l'établissement d'une règle d'action, non l'exécution de l'action elle-même. Comme tout raisonnement pratique, elle vise en effet le jugement, c'est-à-dire l'établissement de la meilleure règle possible, laissant à la décision puis à l'effectuation de la décision le soin de convertir cette règle dans la réalité, ce qui laisse d'ailleurs ouverte la possibilité d'agir en dépit de son meilleur jugement. C'est dans cette faille entre le jugement et l'action que pourrait s'inscrire la problématique de Dupuy concernant la noncrédibilité de la catastrophe: quand bien même on sait, ou quand bien même on peut rationnellement établir qu'il faudrait agir en fonction de la catastrophe possible, on ne le fait pas - l'action ne suit pas le jugement. Mais du coup, la précaution ainsi définie n'est pas non plus à proprement parler une attitude

43 Charles Sanders Peirce, Pragmatisme et pragmaticisme, Euvres I, trad. de Claudine Tiercelin, Paris, éd. du Cerf, 2002, p. 23 (note).

44 Ibid. 
comme peut l'être la prudence: il ne s'agit pas tant de se retenir d'agir par crainte de nuire à autrui ou à soi-même que, encore une fois, d'adopter une technique de raisonnement propre à former un jugement dans des situations d'incertitude.

En revanche, on voit avec quels avantages cette logique de la précaution peut être accompagnée, voire amplifiée par une démocratisation de la communication politique. De ce point de vue, c'est-à-dire si on les met à leur juste place logique, les thèses de Callon, Lascoumes et Barthe gardent toute leur valeur: la démocratie communicationnelle ou dialogique est effectivement le meilleur milieu possible pour un déploiement optimal du principe de précaution. Et cela se comprend aisément, dès lors que l'on saisit le rôle crucial de la pro-duction dans le raisonnement de précaution: puisqu'il s'agit, à divers stades du raisonnement, de formuler des hypothèses exploratoires, de multiplier les points de vue et de décloisonner les savoirs - et ce décloisonnement ne doit pas seulement aller du laboratoire au plein air, mais aussi du plein air au laboratoire -, rien ne peut plus enrichir la production que l'organisation démocratique de la communication entre les acteurs concernés.

\section{LES USAGES DE LA PRÉCAUTION}

En recherchant avec une certaine obstination une définition conceptuellement rigoureuse du PP, nous avons voulu, entre autres choses, nous prémunir contre l'usage inflationniste d'une notion aujourd'hui d'autant plus usitée qu'elle peine à se définir, tout en rassemblant sur elle les indubitables avantages du politiquement correct. Cette invocation tous azimuts du PP suscite à son tour la réflexion et incite à la vigilance. C'est là-dessus que j'aimerais conclure.

1/ Il est possible, comme cela a été noté en introduction, que le PP ait effectivement une importance sociétale, puisqu'il entend réguler une rationalité instrumentale qu'une certaine logique de la modernité voulait simplement voir abandonnée à elle-même. De ce point de vue, le PP peut apparaître comme le signe d'une volonté de réappropriation par l'homme du système des activités instrumentales devenues autonomes, donc menaçantes. L'outil de cette réappropriation est prioritairement la rationalité politique, puisque c'est elle qui entend, en quelque sorte, chapeauter par le PP des activités qui autrement n'en auraient cure - c'est la raison pour laquelle, encore une fois, le PP se trouve effectivement dans la proximité d'un principe démocratique, sans toutefois se confondre avec lui. Mais en même temps, il se pourrait que petit à petit, le $\mathrm{PP}$ s'autonomise à son tour, hypnotisant la rationalité politique au point de faire de l'évaluation des risques le seul critère de décision légitime, comme si les risques étaient le seul aspect sous lequel il faille évaluer une situation d'incertitude. Nous connaissons la «société du risque», mais celle-ci a tôt fait de produire une idéologie du risque qui peut se donner la bonne conscience du PP tout en soustrayant les décideurs à leur responsabilité politique - comme si évaluer, calculer, soupeser des risques était l'ultima ratio, le critère ultime de la décision politique. Il y a donc un usage scientiste du PP en politique qui risque d'en faire, plutôt que le contrepoids, le symptôme de la civilisation technologique qui a été son contexte d'émergence. 
Un exemple: si, conformément à un usage rigoureux du $\mathrm{PP}$, on arrivait à montrer que le clonage reproductif humain, une fois devenu techniquement possible, ne représentait aucun danger scientifiquement repérable d'aucune sorte, et ne serait plus qu'un moyen de reproduction artificielle parmi d'autres, il nous serait encore loisible de prendre la décision politique de l'interdire - non pas au nom des risques encourus, mais au nom de la compréhension que nous avons de nous-mêmes. Nous pourrions dire: ne voulons pas un monde de clones, c'est-àdire un monde où les parents ne désireraient pas un enfant, mais tel enfant, ou cet enfant; nous ne voulons pas d'un tel monde, non parce qu'il nous fait courir des risques, mais parce qu'il bouscule nos représentations les plus fondamentales quant à ce qu'est l'altérité des nouveau-nés. Ce n'est donc pas par précaution que l'on refuserait le clonage, mais par la volonté de construire un monde qui soit à l'image de ce que nous voulons qu'il soit. Si l'innocuité du clonage était prouvée, le PP nous le laisserait faire; mais précisément, la précaution n'est pas le dernier mot de l'affaire. Lorsque le PP a fait son travail - et il faut, assurément, qu'il le fasse -, il cède la place aux vraies questions, qui ne sont pas les questions de risques, mais les questions de choix politiques, et, le cas échéant, de choix culturels ou civilisationnels. Or, dans notre contexte d'incertitude, le PP menace de devenir le cache-sexe de l'irréflexion politique.

2/ Puisque le PP se prête si facilement à un usage scientiste qui en fait le nouveau symptôme d'un positivisme politique, on peut se demander s'il est l'outil adéquat à l'émergence d'une authentique politique de la nature - si tant est bien sûr qu'une telle politique doive voir le jour. Mais à supposer qu'elle le doive, ses défenseurs tiennent-ils avec le PP un outil conceptuel adéquat à leurs propres fins? Cela dépend bien sûr de la hauteur de l'exigence que l'on assigne à ce qu'on appelle une politique de la nature. Mais on peut tout de même réfléchir à ceci. Dans son Principe de responsabilité, Hans Jonas inscrivait l'ensemble de sa démarche à la fois éthique et métaphysique dans le vide éthique laissé par l'Aufklärung: "C'est la question de savoir si sans le rétablissement de la catégorie du sacré qui a été détruite de fond en comble par l'Aufklärung scientifique nous pouvons avoir une éthique capable d'entraver les pouvoirs extrêmes que nous possédons aujourd'hui et que nous sommes presque forcés d'acquérir et de mettre constamment en œuvre $»^{45}$. D'où l'idée, chez lui, non seulement d'une heuristique de la peur (tant décriée, si peu comprise), mais, presque, d'une religion de la peur: «Par rapport aux effets qui nous menacent encore immédiatement nous-mêmes et que nous subissons encore nous-mêmes, la peur, qui tant de fois est le meilleur substitut de la vertu et de la sagesse véritable, peut jouer ce rôle; mais ce moyen échoue face aux perspectives plus lointaines qui importent principalement ici, d'autant plus que la plupart du temps la modestie des débuts semble innocente. Seule la crainte de porter atteinte à quelque chose de sacré est à l'abri des calculs de la peur et de la consolation tirée du caractère incertain des conséquences encore lointaines. Mais une religion absente ne saurait délivrer l'éthique de sa tâche; et alors qu'on peut dire de la première qu'elle existe ou n'existe pas comme fait exerçant une influence déterminante sur les hommes, il faut dire de l'éthique

45 PR 45 
qu'elle doit exister. Elle doit exister parce que les hommes agissent et l'éthique est là pour ordonner les actions et pour réguler le pouvoir d'agir " ${ }^{46}$. Répétons: "Seule la crainte de porter atteinte à quelque chose de sacré est à l'abri des calculs de la peur...». Tout se passe chez Jonas comme si la raison calculatrice n'était pas à la hauteur de la tâche préservatrice de l'humain qui est désormais celle des humains, comme si seul un autre que l'humaine raison pouvait protéger la raison contre elle-même - écho lointain, peut-être, à la fameuse phrase de Dostö̈evski proclamant que «Si Dieu n'existe pas, tout est permis ». En suggérant ainsi que le salut se trouve dans le sacré, Jonas entend résoudre le même problème que celui auquel Dupuy s'acharne à trouver une solution rationnelle: comment la raison humaine peut-elle mobiliser la volonté contre ses propres pouvoirs? Jonas, pour ce faire, resacralise la nature, imposant à la raison un Autre qui la limite, Dupuy pousse la rationalité jusque dans ses derniers retranchements - au-delà d'ailleurs, peut-être, de ce qu'elle peut supporter. Limitation contre auto-limitation: le débat ne porte que sur les moyens. On pourrait en imaginer d'autres, moins subtils, mais qui visent la même fin, comme d'accorder des droits à la nature, ou de passer un «contrat naturel»... Il n'empêche que ce débat de fond rejaillit directement sur le principe de précaution: car s'il n'est, comme nous avons essayé de le montrer, qu'une manière de raisonner, si, comme nous l'a appris l'école prudentielle, cette manière de raisonner est essentiellement liée au calcul des conséquences, alors on voit bien qu'il n'est lui-même qu'un instrument au service de la raison instrumentale. Et si l'on ajoute - comme nous le suggère le miroir grossissant de la pensée catastrophiste - que dans le cas de la catastrophe précisément, la conséquence envisageable, toute catastrophique qu'elle soit, est néanmoins trop éloignée pour pouvoir mobiliser la volonté, alors il devient évident que par lui-même, le PP, lié par son concept même à la raison calculatrice, n'est pas à la hauteur d'une véritable pensée de la nature. Entièrement situé sous le paradigme instrumental du calcul des conséquences, le PP ne vise qu'à gérer rationnellement les incertitudes que la pensée instrumentale a elle-même produites. Eloigné par lui-même de toute idée de respect, il n'est qu'une aide à la raison instrumentale pour la prévenir de ses propres excès - un correctif au service de la cause qu'elle corrige*.

46 PR 45.

* La première version de ce texte a été publiée, avec le titre «Pourquoi avons-nous besoin du raisonnement de précaution?» (pp. 139-162) dans le fascicule consacré au thème des «Risques et précaution» du numéro août-septembre 2003 de la revue «Esprit». Il a été placé en annexe («La logique du raisonnement de précaution», pp. 149-192) dans mon livre «Je est un clone. L'éthique à l'épreuve des biotechnologies », Paris, Editions du Seuil, 2004. 